\title{
Statistics of Yang-Mills Solitons
}

\author{
John L. Friedman ${ }^{1, \star}$ and Rafael D. Sorkin ${ }^{2}$ \\ 1 Physics Department, University of Wisconsin-Milwaukee, Milwaukee, WI 53201, USA \\ 2 Institute for Advanced Study, Princeton, NJ 08540 and \\ Center for Theoretical Physics, University of Maryland, College Park, MD 20742, USA
}

\begin{abstract}
We determine the statistics and the spin of isolated Yang-Mills monopoles in eigenstates of their electric and magnetic charge. Exchange of solitons is defined using the translation operator of a companion paper; and under exchange, state vectors representing $N$ identical solitons change sign precisely when the angular momentum of each soliton is half-integral.
\end{abstract}

\section{Introduction}

The symmetries of classical mechanics can be understood as expressing a freedom in the relation between a subsystem and its environment. When this environment is neutral in some neighborhood of the subsystem - when, for example, the forces due to external matter are small - it makes sense to translate or rotate the subsystem without altering its internal state. Then the invariance of the total system's action under such changes implies the conservation of the momentum and angular-momentum of the subsystem.

In gauge theories, an invariance of the vacuum (neutral environment) under some global gauge-transformation implies a further freedom, one which is exercised by applying the transformation to the subsystem but not to the environment, just as one rotates a subsystem by applying "rotation" to it but not to the objects around it. Here again symmetry leads to conservation laws, for example to the conservation of the electric charge of the subsystem in theories with an unbroken phase-invariance.

When such "internal" gauge-symmetries are present alongside spacetime ones any symmetry operation can be composed with an "internal" one without affecting its geometrical content. In particular the operation of exchanging a pair of indistinguishable subsystems (which might be single particles) via a sequence of translations through the vacuum is ambiguous until one specifies which of the gauge-equivalent versions of translation is to be used at each stage.

* Supported in part by the National Science Foundation 
For identical particles with only an overall U(1) freedom one can resolve this ambiguity locally by requiring that infinitesimal translations in different directions commute. This yields a physically appropriate exchange, but one whose result need not be the identity operation. When it is not, the particles (or subsystems) in question are not acting like bosons, and this can happen even in a theory in which the fundamental fields are tensors quantized via canonical commutation relations.

The prototype of such an "anomalously fermionic" subsystem is what we will call a "Dirac-dyon," a system consisting of one particle with electric charge $Q_{E}$ and one with magnetic charge $Q_{B}$. A Dirac-dyon whose constituents are bosons has half-integral angular momentum when the angular momentum $Q_{E} Q_{B}$ due to the electric and magnetic coulomb fields is half-integral [1]. In a Yang-Mills-Higgs field theory, the 't Hooft-Polyakov monopole [2] is an extended object that looks from afar like a magnetically charged particle; and by adding a charged boson field to the extended monopole, Jackiw and Rebbi and Hasenfratz and 't Hooft [3] obtained an extended dyon in which the angular momentum is again half-integral when the product $Q_{E} Q_{B}$ of the total electric and magnetic charge operators has half-integral eigenvalues.

Dyons constructed from point particles and having half-integral angular momentum exhibit fermi statistics. When the separation between two identical dyons is large compared to the size of each dyon and one interchanges the dyons by a simultaneous translation of each to the position of the other, the gauge transformation that accompanies the translation of charged particles in a magnetic field changes the sign of the wave function $[4,5]$. More formally, the configuration space of $N$ separated dyons can be decomposed into a product of $N$ bundles, the configuration spaces of the individual dyons. And the spin-statistics relation is recovered for the unique dyon interchange that corresponds to this decomposition.

One expects that in the asymptotic limit, charged Yang-Mills-Higgs monopoles will behave like separated Dirac-dyons and that a spin-statistics relation will hold here as well. We find that this expectation is in fact correct. However, in the Dirac quantization condition $Q_{E} Q_{B}=\frac{n}{2}$, the electric charge is replaced in the $\theta$-vacuum theory by a linear combination $q=Q_{E}+\frac{e^{2} \theta}{2 \pi} Q_{B}$ and monopoles are bosons or fermions when $q Q_{B}$ is integral or half-integral, respectively.

By means of the monopole translation defined in the companion paper (Paper I), we establish in Sects. III and IV that dyons with half-integral spin are fermions and dyons with integral spin are bosons ${ }^{1}$. (Dyon $\equiv$ monopole in an eigenstate of electric and magnetic charge.) We show first that if one interchanges two identical distant dyons by simultaneously translating each to the position of

1 The statistics of Yang-Mills dyons was previously discussed by Ezawa [6] who claims to show the existence of dyon creation operators satisfying anticommutation relations. However, we believe that the key equation, (2.9), of that paper is false because the element $\Omega(\theta=\pi, \phi)$ of SU(2) appearing there does not have the value -1 (instead, its square $\Omega(\theta=2 \pi, \phi)$ is -1 ). Thus what is really shown is not that the (gauge-dependent) operators denoted " $\psi_{a}(X)$ " and " $\psi_{b}(Y)$ " in that paper anticommute, but that their anticommutator does not vanish 
the other, the state vector changes sign precisely when each dyon has half-integral angular momentum. This suggests that the space of state vectors describing $N$ identically charged dyons ought to decompose as a properly symmetrized tensor product of one-dyon state spaces. In Sect. IV we verify this for state functionals $\psi$ defined on an "asymptotic" configuration space representing the $R / l \rightarrow 0$ limit of $N$ monopoles with arbitrary internal configurations. In their interaction with each other identical dyons are thus fermions or bosons in the strongest possible ${ }^{2}$ sense.

Our result is reminiscent of much earlier work by Finkelstein and Rubinstein [7], who establish a spin-statistics theorem for the interchange of "kinks." Beginning with a non-simply connected space of field configurations, they show that a theory whose configuration space is the associated covering space admits double-valued representations of the rotation group precisely when an interchange of kinks by translation can be nontrivially represented. However, we have been unable to interpret our results as a consequence of their general arguments. In the first place the effective configuration space is for them larger than the space of field configurations, whereas in a gauge-theory it is smaller. Moreover, the FinkelsteinRubinstein definition of vacuum does not apply here because the fields (in particular the Higgs field) are not constant in the Higgs vacuum that separates the monopoles. Nor, for this reason, can monopole exchange be accomplished via the naive field translations they use to define kink exchange; instead, the naive translation $\mathscr{F}(r) \rightarrow \mathscr{F}(r-a)$ is accompanied by a gauge transformation, as was discussed in Paper I. Finally, their basic lemma that kink exchange is homotopic to kink-rotation is vacuous here because the space of field configurations is simply connected $^{3}$.

\section{Formalism}

Our notation and definitions will conform to those of Paper I. In particular, $\mathscr{F}$ will denote a triplet $\left(A^{a}{ }_{i}, \Phi^{a}, \sigma^{I}\right)$ of fields on $\mathbb{R}^{3}$, an $\mathrm{SU}(2)$ Yang-Mills field (in a temporal gauge), a Higgs field, and a spinless isodoublet $\sigma^{I}$. Here $i, j, \ldots$ are spatial indices, $a, b, \ldots$ are Lie algebra $\left(\mathrm{SO}_{3}\right)$ indices, and $I, J, \ldots$ are indices in the space of the two-component isospinor $\sigma^{I}$. By a configuration $[\mathscr{F}]$ is meant an equivalence class of fields, where $[\tilde{F}]=\left[\mathscr{F}^{\prime}\right] \Leftrightarrow \mathscr{F}^{\prime}=\underset{\sim}{\mathscr{F}}$, where $\bigcup_{\sim}$ is the gauge transformation associated with a map $U: \mathbb{R}^{3} \rightarrow \mathrm{SU}(2)$ that is asymptotically the identity. We denote by $\mathscr{C}^{N}{ }_{m}$ the space of configurations $[\mathscr{F}]$ which describe $N$ distinct monopoles of magnetic charge $g=-\frac{m}{e}$ with fixed asymptotic gauge. Precisely, $[\mathscr{F}] \in \mathscr{C}^{N}{ }_{m}$ if the following conditions are satisfied:

$$
\begin{gathered}
A^{a}{ }_{i}=\varepsilon^{a b c} \partial_{i} \Phi_{b} \Phi_{c}+O\left(r^{-2}\right), \\
\Phi^{a}=\hat{x}^{a} \sin \theta \cos N m \phi+\hat{y}^{a} \sin \theta \sin N m \phi+\hat{z}^{a} \cos \theta+O\left(r^{-1}\right) ;
\end{gathered}
$$

2 What is not possible is to have a tensor-product decomposition when unlike dyons are present

3 By restricting consideration to the subspace $\mathscr{C}^{N}$ of $N$-monopole fields we could get a topologically non-trivial configuration space. But the homotopy in [7] converting exchange to rotation proceeds via the creation and annihilation of kink-antikink pairs and thus would not be expected to have an analogue remaining within the $N$-monopole sector 
there is some $R>0$ and $N$ disjoint balls $\beta_{\alpha}$ of radius $R$ and centers $o_{\alpha}$ for which $\mathscr{F}$ is Higgs vacuum outside $\bigcup_{\alpha} \beta_{\alpha}$ - that is,

$$
F_{i j}^{a}=e \Phi^{a} F_{i j}, D_{i} \Phi^{a}=0, \text { and } \quad \sigma^{I}=0, r_{\alpha}>R,
$$

where $r_{\alpha}^{i}=r^{i}-o_{\alpha}^{i} ; F_{i j}$ satisfies

$$
F_{i j}=\sum_{\alpha} \stackrel{\alpha}{F}_{i j}=\sum_{\alpha}-\frac{m}{e} \frac{\varepsilon_{i j k} r_{\alpha}{ }^{k}}{r_{\alpha}{ }^{3}} ;
$$

and $\Phi^{a}$ vanishes at each point $o_{\alpha}$ and nowhere else. The space $\mathscr{C}^{N}{ }_{m}$ is a fiber bundle with fibers isomorphic to the $N$-fold product $\mathrm{U}(1) \times \ldots \times \mathrm{U}(1)$, and two configurations $[\mathscr{F}],\left[\mathscr{F}^{\prime}\right] \in \mathscr{C}^{N}{ }_{m}$ belong to the same fiber if they have the same monopole locations and internal configurations, i.e., if for some phases $u_{\alpha}\left(\chi_{\alpha}\right)=e^{i \chi_{\alpha}}$ the associated $\mathrm{U}(1)$-rotations $\underline{\sim}_{\alpha}\left(\chi_{\alpha}\right)$ of the monopoles at $\left\{o_{\alpha}\right\}$ transform $\mathscr{F}$ to $\mathscr{F}^{\prime}$ :

$$
\left[\mathscr{F}^{\prime}\right]=u_{1}\left(\chi_{1}\right) \ldots u_{N}\left(\chi_{N}\right)[\mathscr{F}]
$$

where

$$
\underline{\sim}_{\alpha}\left(\chi_{\alpha}\right)= \begin{cases}{\underset{\sim}{\alpha}}_{\alpha}\left(\chi_{\alpha}\right) \mathscr{F}, & r_{\alpha}<R+\varepsilon \\ \mathscr{F}, & \text { elsewhere, }\end{cases}
$$

with $U_{\alpha}\left(\chi_{\alpha}\right)=\exp \left(i \chi_{\alpha} f \Phi^{a} \tau_{a}\right), f$ any smooth function equal to 1 outside $r_{\alpha}=R+\varepsilon$.

The corresponding $N$-dyon state space of functions $\psi$ on $\mathscr{C}^{N}{ }_{m}$ for which each monopole has electric charge $n \frac{e}{2}$ is denoted $\mathscr{H}_{m n}^{N}$, and $\psi \in \mathscr{H}_{m n}^{N}$ if $\psi$ is homogeneous of degree $n$ with respect to each $\mathrm{U}(1)$ :

$$
\psi\left(u_{\alpha}[\mathscr{F}]\right)=u_{\alpha}^{n} \psi([\mathscr{F}]) .
$$

Operators on $\mathscr{C}^{N}{ }_{m}$ corresponding to infinitesimal translations and rotations of individual monopoles were defined in Paper I, Eqs. I(58) and I(59). The change in $[\mathscr{F}]$ arising from the infinitesimal translation of a monopole at $o_{\alpha}$ along the constant vector field $n^{i}$ is written $\stackrel{\alpha}{\delta}_{n}[\mathscr{F}]$, and the change arising from an infinitesimal rotation along a rotational vector field $\xi^{i}:=\varepsilon^{i}{ }_{j k} n^{j} r_{\alpha}{ }^{k}$ is written $\stackrel{\alpha}{\xi}_{\xi}^{\alpha}[\mathscr{F}]$.

The commutator of two translations is [Eq. I(54a)]

$$
\left[\stackrel{\alpha}{\delta}_{n}, \stackrel{\alpha}{\delta}_{\bar{n}}\right]=-\mathscr{U}_{\alpha}\left(\sum_{\alpha^{\prime}}{\stackrel{\alpha^{\prime}}{F}}_{j k}\left(o_{\alpha}\right) n^{j} \bar{n}^{k}\right)+\sum_{\alpha^{\prime}} \mathscr{U}_{\alpha^{\prime}}\left(\stackrel{\alpha}{F}_{j k}\left(o_{\alpha^{\prime}}\right) n^{j} \bar{n}^{k}\right)
$$

where $\mathscr{U}_{\alpha}(\chi)$ denotes an infinitesimal U(1)-rotation; while single monopole translations commute with overall translations of the field $\mathscr{F}$ (denoted $\delta_{n}[\mathscr{F}]$ ):

$$
\left[\delta_{n}, \stackrel{\alpha}{\delta}_{\bar{n}}\right]=0
$$

\section{Exchange of Monopoles by Asymptotic Translation}

We saw in Paper I that an $N$-monopole configuration $[\mathscr{F}] \in \mathscr{C}^{N}$ is not fully determined by the locations and internal configurations of the monopoles, that 
there remains for each monopole a further phase degree of freedom, expressing how that monopole fits into its environment. These extra U(1) degrees of freedom make possible the "anomalous" appearance of dyons of half-integral spin and, as we shall see, fermi statistics in a theory constructed only from vector and scalar fields.

More precisely, we will show (Theorem 2, in Sect. IV) that in the asymptotic limit of large monopole separation, the Hilbert space $\mathscr{H}_{m, n}^{N}$ has a decomposition in terms of 1-dyon Hilbert spaces

$$
\mathscr{H}_{m, n}^{N} \subset \mathscr{H}_{m, n}^{1} \otimes \ldots \otimes \mathscr{H}_{m, n}^{1},
$$

where $\mathscr{H}_{m, n}^{1}$ is itself the tensor product of spaces representing the internal and external degrees of freedom of a single dyon of charge $n \frac{e}{2}$,

$$
\mathscr{H}_{m, n}^{1}=L^{2}\left(R^{3}\right) \otimes \mathscr{H}_{m, n}^{1_{\mathrm{int}}}
$$

When the product $m n$ is even, $\mathscr{H}_{m, n}^{N}$ will be the symmetric subspace of $\mathscr{H}_{m, n}^{1} \otimes \ldots \otimes \mathscr{H}_{m, n}^{1}$, and when $m n$ is odd, the antisymmetric subspace.

To begin with, we show that dyon exchange realized via the asymptotic translations defined in Paper I is equivalent to a $U(1)$ rotation by $m \pi$ of one of the dyons. Together with our earlier result that the $2 \pi$ spatial rotation of a single monopole is also equivalent to a $\mathrm{U}(1)$ rotation by $m \pi$, this provides a heuristic spin-statistics relation, reminiscent of that in Finkelstein and Rubinstein [7].

We then carry out (in Sect. IV) the above mentioned decomposition of $\mathscr{H}_{m, n}^{N}$, which allows one to speak of dyons as particles with well-defined statistics. Here again we employ the notion of asymptotic translation, this time in the guise of a connection on a bundle of asymptotic $N$-monopole configurations. In this discussion, the mathematics is very close to that used in [5] to derive a spinstatistics theorem for dyons composed of point electric and magnetic charges. Only there the relevant $U(1)$ degree of freedom had been introduced ad hoc in order to get an action principle for the system of elementary point charges. Here it arises from the phase ambiguity automatically associated with each Yang-Mills monopole, or, in other language, from the fact that $\mathscr{C}^{N}$ is a bundle over the space $\mathscr{M}^{N}$ of monopole locations and internal configurations. It is ultimately the structure of this bundle that determines dyon statistics, and in Sect. IV the asymptotic translations will serve simply as a convenient means of deducing this structure.

Were one concerned only with SU(2) theories, parts of Sect. IV would merely be duplicating the derivation of the minus sign accomplished more suggestively in the present section. However, we have chosen to present an ab initio derivation, because the method adopted in Sect. IV should carry over without change to any theory in which - as here - the spontaneous symmetry breaking leaves one with an effective U(1) gauge group.

Let us consider a state vector $\psi$ in the two dyon state space $\mathscr{H}_{m n}^{2}$ and suppose that $\psi$ has support on configurations in $\mathscr{C}_{m}^{2}$ for which the internal configurations of the monopoles are identical. We will exchange the monopoles of such a configuration by translating each of them to the position of the other, in accordance 
with Eqs. I(53) - i.e., in the approximation that each is translated in a constant coulomb field due to the other monopole. After the exchange has been completed, the final configuration will differ from the original only by $U(1)$ rotations of the monopoles; and we shall see that the effect of these $U(1)$ rotations is to change the sign of $\psi(\mathscr{F})$ precisely when $m n$ is odd.

Denote by $\beta_{ \pm}$the balls of centers $o_{ \pm}$and radius $R$ containing the two monopoles; let $l$ denote the monopole separation $\left|o_{+}-o_{-}\right|$. We will consider the exchange $E$ that results from translating each monopole along an arbitrary path to the position of the other monopole, in accordance with Eq. I(53). With the help of three lemmas we can prove the main result of this section.

Theorem 1. Let $\psi \in \mathscr{H}_{m n}^{2}$ have support on configurations [F्F] for which the monopoles have the same internal configuration. Then to leading order in $\lambda \equiv \frac{R}{l}$

$$
E \psi=(-1)^{m n} \psi
$$

Without altering the asymptotic conditions (1), we can pick $r=0$ to be midpoint of the monopoles. Let $n^{i}$ be a unit vector perpendicular to the plane of the semicircles $o_{ \pm}(t)$. Then $\xi^{i}=\varepsilon^{i}{ }_{j k} r^{j} n^{k}$ is the rotational symmetry vector tangent to $o_{ \pm}(t)$, and the corresponding rotational symmetry vectors that generate rotations about the $n$-axis through $o_{ \pm}$are

$$
\zeta_{ \pm}^{i}=\xi^{i}-\xi^{i}\left(o_{ \pm}(t)\right) \equiv \xi^{i} \mp \xi_{o}^{i}(t) .
$$

Lemma 1. The simultaneous infinitesimal translation of $\beta_{+}$by $\xi_{o}^{i}$ and $\beta_{-} b y-\xi_{o}^{i}$ is equivalent to an infinitesimal (counterclockwise) rotation of $\mathscr{F}$ by $\xi^{i}$ together with infinitesimal (clockwise) rotations of each monopole by $-\zeta_{ \pm}^{i}$, respectively.

Proof of Lemma 1. We need to verify that

$$
\left(\delta_{\xi}-\delta_{\zeta_{+}}-\delta_{\zeta_{-}}\right)=\left(\stackrel{+}{\delta}_{\xi_{o}}-\bar{\delta}_{\xi_{o}}\right) \cdot(1+O(\lambda)),
$$

where ${\stackrel{+}{\delta_{\xi_{o}}}}_{\text {and }} \bar{\delta}_{\xi_{o}}$ are the infinitesimal translations by $\xi_{o}^{i}$ of $\beta_{+}$and $\beta_{-}$respectively. First, because $\hat{r} \cdot n=\frac{1}{2}\left(\hat{r}_{+} \cdot n+\hat{r}_{-} \cdot n\right)+O\left(r^{-2}\right), \delta_{\xi}$ as given by Eq. I(32) is equivalent to

$$
\delta_{\xi}=-\xi^{j} \mathscr{D}_{j}-\Delta\left[m\left(\hat{r}_{+} \cdot n+\hat{r}_{-} \cdot n\right) \Phi^{a}\right] .
$$

Equation $\mathrm{I}(55)$ then implies

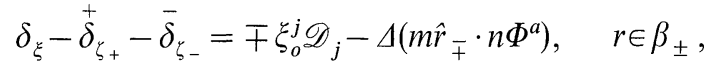

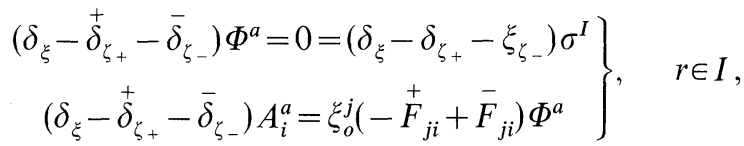

where in the last equality we used $F_{i j}^{a}=\left(\stackrel{+}{F}_{i j}+\bar{F}_{i j}\right) \Phi^{a}, r \in I$.

On the other hand, from Eqs. I(53) we have (to leading order)

$$
\stackrel{+}{\delta}_{\xi_{o}}-\bar{\delta}_{\xi_{o}}=\mp \xi_{o}^{j} \mathscr{D}_{j} \mp \Delta\left(2 \bar{F}_{j k}\left(o_{+}\right) \xi_{o}^{j} r^{k}\right), \quad r \in \beta_{ \pm},
$$




$$
\left.\begin{array}{l}
\left(\stackrel{+}{\delta}_{\xi_{o}}-\bar{\delta}_{\xi_{o}}\right) \Phi^{a}=0=\left(\stackrel{+}{\delta}_{\xi_{o}}-\bar{\delta}_{\xi_{o}}\right) \sigma^{I} \\
\left(\stackrel{+}{\delta}_{\xi_{o}}-\bar{\delta}_{\xi_{o}}\right) \mathrm{A}_{i}^{a}=\xi_{o}^{j}\left(-\stackrel{+}{F}_{j i}+\bar{F}_{j i}\right)
\end{array}\right\}, \quad r \in I .
$$

Equations (10) and (11) differ only in the arguments of $\Delta$ in Eqs. (10a) and (11a). To compare them, note that for $r \in \beta_{+}$,

$$
\bar{F}_{j k}\left(o_{+}\right) \xi_{o}^{j} r^{k}=\frac{m}{\left|2 o_{+}\right|^{2}} \hat{o}_{+} \times \xi_{o} \cdot r=\frac{m}{4\left|o_{+}\right|} n \cdot r=\frac{m}{2} \hat{r}_{-} \cdot n(1+O(\lambda))
$$

using $\left|\xi_{0}\right|=\left|o_{+}\right|,|r|=2\left|o_{+}\right|(1+O(\lambda)), r \in \beta_{+}$. Similarly, for $r \in \beta_{-}$

$$
\bar{F}_{j k}\left(o_{+}\right) \xi_{o}^{j} r^{k}=-\frac{m}{2} \hat{r}_{+} \cdot n(1+O(\lambda))
$$

Thus, as claimed, Eqs. (10) and (11) differ only by $O(\lambda)$.

Lemma 2. Let $[\overline{\mathscr{F}}] \in \mathscr{C}_{m}^{2}$ be a configuration whose monopoles have the same internal configuration and are centered on the $y$-axis. Then there is a representative $\mathscr{F} \in[\overline{\mathscr{F}}]$ for which

$$
\mathscr{F}(r)=\hat{R}(\pi \hat{z}) \mathscr{F}(r), \quad r \in I .
$$

In Eq. (12), $\hat{R}(\pi \hat{z})$ is the "naive" $\pi$-rotation $\mathrm{I}(35)$ of $\mathscr{F}$ as a vector and two scalars about the $\hat{z}$-axis through the midpoint of the monopoles.

Proof of Lemma 2. By a gauge transformation in $\mathscr{G}^{\infty}$, one can rotate $\Phi^{a}$ for $r_{ \pm}>\varepsilon$ to any other (smooth) isovector field of the same magnitude and the same Euler indices at $o_{ \pm}$. In particular, let

$$
\Phi^{a}=R\left[m\left(\phi_{ \pm} \pm \frac{\pi}{2}\right) \hat{z}\right]_{b}^{a} \hat{x}^{b} \sin \theta_{ \pm}+\hat{z}^{a} \cos \theta_{ \pm}, \quad R \leqq r_{ \pm} \leqq R+2 \varepsilon,
$$

and, in accordance with (10),

$$
\Phi^{a}=R(2 m \phi \hat{z})^{a}{ }_{b} \hat{x}^{b} \sin \theta+\hat{z}^{a} \cos \theta, \quad r>2 l,
$$

where $\left(r_{ \pm}, \theta_{ \pm}, \phi_{ \pm}\right)$are spherical coordinates about $o_{ \pm}$.

Then $\Phi^{a}\left[R(\pi \hat{z})^{-1} r\right]=\Phi^{a}(r)$ outside $r=2 l$ and in the shells about $\beta_{ \pm}$, and one can smoothly interpolate a field $\Phi^{a}$ in the intervening region with this symmetry under $\pi$-rotation. Figure 1a shows an example of such a field for $m=1$ monopoles.

Now in the intermonopole region $I$ one can find $A_{i}^{a}$ with the symmetries of $\Phi^{a}$ as follows. In $I, D_{i} \Phi^{a}=0$ implies that there is some $\bar{A}_{i}^{i}$ for which

$$
A^{a}{ }_{i}=\varepsilon_{b c}^{a} \partial_{i} \Phi^{b} \Phi^{c}+\Phi^{a} \bar{A}_{i}
$$

and

$$
F_{i j}^{a}=-\varepsilon_{b c}^{a} \partial_{i} \Phi^{b} \partial_{j} \Phi^{c}+\Phi^{a}\left(\partial_{i} \bar{A}_{j}-\partial_{j} \bar{A}_{i}\right) .
$$

Since $F^{a}{ }_{i j}$ and $\Phi^{a}$ are invariant under $\Phi^{a}$, Eq. (14) holds with $\bar{A}_{i}(r)$ replaced by $\hat{R} \bar{A}_{i}(r)=R_{i}{ }^{j} \bar{A}_{j}\left(R^{-1} r\right)$. Then if $2 A_{i}:=\bar{A}_{i}+\hat{R} \bar{A}_{i}$, a vector potential for $F^{a}{ }_{i j}$ invariant under $\hat{R}(\pi \hat{z})$ on $I$ is

$$
A^{a}{ }_{i}=\varepsilon_{b c}^{a} \partial_{i} \Phi^{b} \Phi^{c}+\Phi^{a} A_{i} .
$$



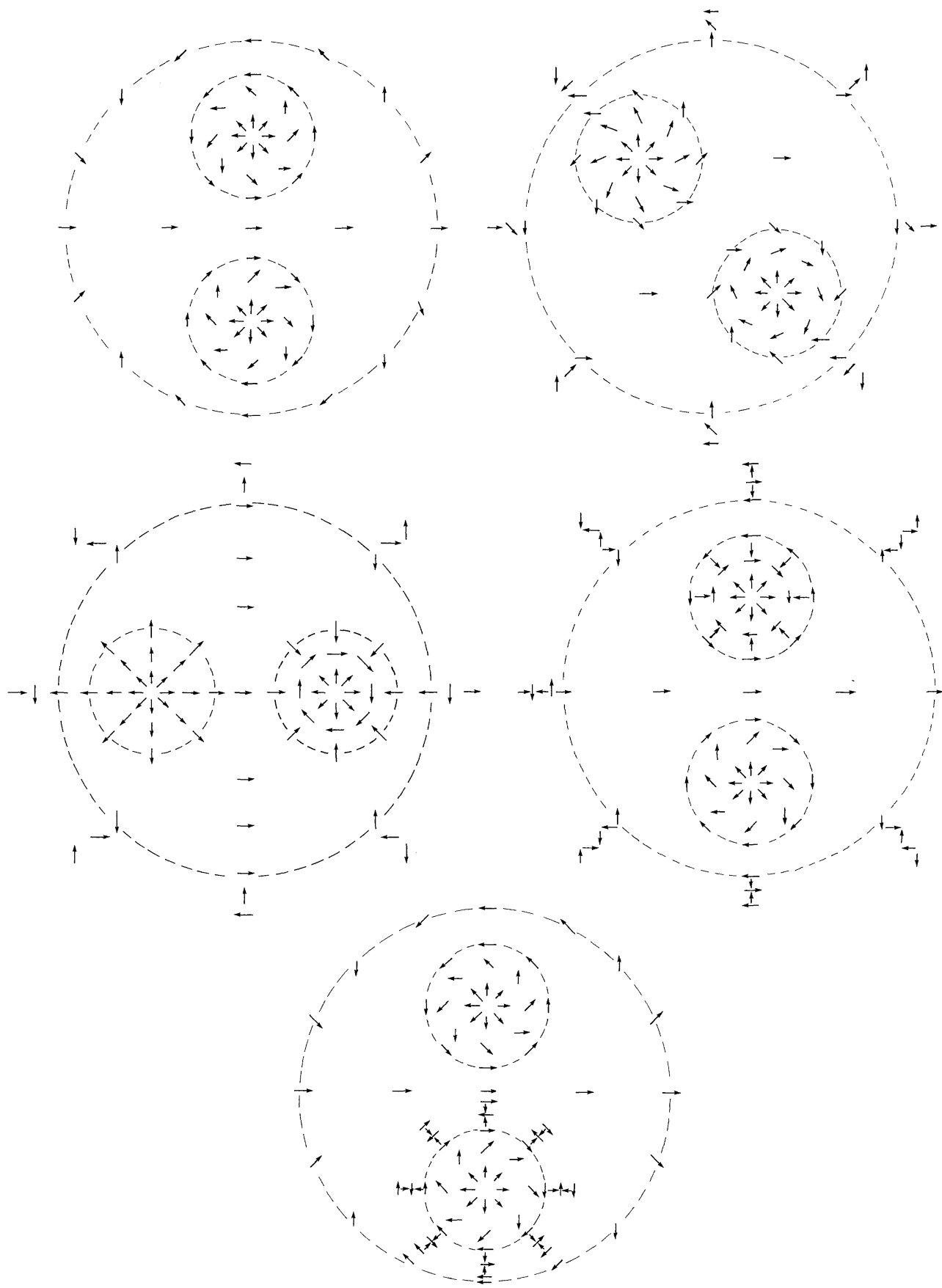

Fig. 1. The Higgs field in the $x-y$ plane of two $m=1$ monopoles is shown at stages of the translation $\mathscr{F}_{t}$ of each monopole to the position of the other. Figures (a)-(d) illustrate $\mathscr{F}_{t}$ of Eq. (24), except that here we have fixed $\Phi^{a}$ near the center of each monopole: Thus $U\left(-t \hat{z}^{a}\right)$ in $(24)$ has been replaced by the $\mathscr{G}^{\infty}$-equivalent transformation $U\left(-F\left(r_{ \pm}\right) t \hat{z}^{a}\right)$, where $F(r)$ increases from 0 to 1 for $0 \leqq r \leqq R$. Between (d) and (e), the $2 \pi$ twist outside $r=2 l$ has been pushed inwards, untwisting $\Phi^{a}$ in the upper monopole, but leaving a $2 \pi$-twist around the lower one. The equivalence between $E \mathscr{F}$ and $R(2 \pi) \mathscr{F}$ is visible as a homotopic equivalence in the $x-y$ plane of the Higgs fields that result from exchange and from rotation, when $\Phi^{a}$ is fixed near $o_{ \pm}$and outside $r=2 l+\varepsilon$ 
To extend $\mathscr{F}=\left(A^{a}{ }_{i}, \Phi^{a}, 0\right)$ to $\beta_{ \pm}$, choose any gauge inside that agrees with (15) at the boundaries $\partial \beta_{+}$. The resulting $\mathscr{F}$ satisfies (12) and by Lemma 3.1 differs only by $U(1)$ rotations from $[\overline{\mathscr{F}}]$. Finally, because $U(1)$ rotations of the form (5) with $f \equiv 1$ are the identity for $r_{ \pm} \geqq R$, we can $\mathrm{U}(1)$ rotate $\mathscr{F}$ to make $[\mathscr{F}]=[\overline{\mathscr{F}}]$ and retain the symmetry (12) on $I$.

Corollary. Let $[\overline{\mathscr{F}}]$ be as in Lemma 2. Then there is a field $\mathscr{F}, \mathrm{U}(1)$ related to $\overline{\mathscr{F}}$, satisfying Eqs. (13), and such that

$$
\begin{gathered}
\hat{R}(\pi \hat{z}) \mathscr{F}=\mathscr{F}, \quad r_{ \pm} \geqq R, \\
A^{a}{ }_{i}=\varepsilon^{a}{ }_{b c} \partial_{i} \Phi^{b} \Phi^{c}\left(1+O\left(\lambda^{2}\right)\right), \quad R \leqq r_{ \pm} \leqq R+\varepsilon, \\
\mathscr{F}\left(r+o_{-}\right)=U(m \pi \hat{z}) \mathscr{F}\left(r+o_{+}\right)+O\left(\lambda^{2}\right)\left(R^{-1}, 1,|\sigma|\right), \quad r_{ \pm} \leqq R+\varepsilon .
\end{gathered}
$$

Proof. For $r_{ \pm} \geqq R+2 \varepsilon$, let $\mathscr{F}$ be as in Lemma 2 with $\Phi^{a}$ given by (13). Then

$$
\varepsilon_{b c}^{a} \partial_{i} \Phi^{b} \Phi^{c}+\Phi^{a} \stackrel{ \pm}{A_{i}^{\mathrm{ext}}}
$$

is a vector potential for $F_{i j}^{a}$ in the shells $R \leqq r_{ \pm} \leqq R+2 \varepsilon$, where $\stackrel{ \pm}{A}_{i}^{\text {ext }}$ given by I(41) is a vector potential for $e \stackrel{\bar{F}_{F}^{\mathrm{ext}}}{i j}=e{\stackrel{\mp}{F^{F}}}_{i j}$. Let $A^{a}{ }_{i}$ have the form (17) for $R \leqq r_{ \pm} \leqq R+\varepsilon$ and match it to the $A^{a}{ }_{i}$ of Eq. (15) outside $r_{+}=R+2 \varepsilon$ by setting

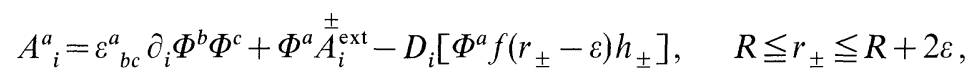

where $\partial_{i} h_{ \pm}=\stackrel{ \pm}{A}_{i}^{\text {ext }}-A_{i}$ and $f\left(r_{ \pm}-\varepsilon\right)$, as in Eq. I(39), rises from 0 to 1 in the shell $R+\varepsilon \leqq r_{+} \leqq R+2 \varepsilon$. Within $\beta_{+}$let $\mathscr{F}$ have any gauge consistent on $\partial \beta_{+}$with Eqs. (13) and (17).

It remains to specify $\mathscr{F}$ in $\beta_{-}$. Because $[\mathscr{F}]_{+}=[\mathscr{F}]_{-}, \mathscr{F}_{-}^{\text {int }} \in[\mathscr{F}]_{-}$, where

$$
\mathscr{F}_{-}^{\text {int }}\left(r+o_{-}\right)=\underset{\sim}{U}(m \pi \hat{z}) \mathscr{F}_{+}^{\text {int }}\left(r+o_{+}\right), \quad r \leqq R ;
$$

moreover, Eqs. (13a) and (18) imply that (19a) holds at $\partial \beta_{-}$. We may therefore specify $\mathscr{F}$ in $\beta$ - by Eq. (19a). Then for $r \leqq R+\varepsilon$,

$$
\left.\begin{array}{rl}
\Phi^{a}\left(r+o_{-}\right) & =R_{b}^{a}(m \pi \hat{z}) \Phi^{b}\left(r+o_{+}\right), \\
\sigma^{I}\left(r+o_{-}\right) & =U(m \pi \hat{z})_{J}^{I} \sigma^{J}\left(r+o_{+}\right), \\
\left.\bar{A}_{i}^{\mathrm{ext}}\right)\left(r+o_{-}\right) & =R_{b}^{a}(m \pi \hat{z})\left(A_{i}^{b}-\Phi^{b} \stackrel{+}{A}_{i}^{+e^{\mathrm{ext}}}\right)\left(r+o_{+}\right) .
\end{array}\right\}
$$

Now for $r_{ \pm} \leqq R+2 \varepsilon,{\stackrel{ \pm}{F^{e x t}}}_{i j}=O\left(\lambda^{2}\right) F_{i j}$ and $A_{i}^{\text {ext }}=O\left(\lambda^{2}\right) \varepsilon_{b c}^{a} \partial_{i} \hat{\Phi}^{b} \hat{\Phi}^{c}$. Then Eqs. $(16 \mathrm{a}-\mathrm{b})$ follow from (18) and the fact that $A_{i}$ and $\Phi^{a}$ are invariant under $\hat{R}(\pi \hat{z})$; and (16c) follows immediately from Eqs. (19).

Lemma 3. Let $\mathscr{F}$ with $[\mathscr{F}] \in \mathscr{C}_{m}^{2}$ satisfy Eqs. (13) and (16). Let E[Fु be the exchange that results from parallel transporting each monopole counterclockwise to the position of the other. Then, to leading order in $\lambda$,

$$
E[\mathscr{F}]=\underset{\sim}{R}(2 \pi)[\mathscr{F}],
$$

where $R_{-}$is a $2 \pi$ rotation of $\beta_{-}$.

That is, when $[\mathscr{F}]$ has the particular $\mathrm{U}(1)$ relation between its monopoles that results from our imposed symmetries, monopole exchange is equivalent to a 
$2 \pi$-rotation of a single monopole. This is illustrated for the Higgs field of two $m=1$ monopoles in Figs. 1(a)-(d).

Proof of Lemma 3. As in (9), let

$$
\xi_{o}^{i}(t)=\xi^{i}\left(o_{+}(t)\right), \quad \zeta_{ \pm}^{i}(t)=\xi^{i} \mp \xi_{o}^{i}(t) .
$$

From Lemma $4.1, E \mathscr{F}=\mathscr{F}_{t=\pi}$, where $\mathscr{F}_{t=0}=\mathscr{F}$ and

$$
\frac{d}{d t} \mathscr{F}_{t}=\left[\delta_{\xi}-\delta_{\zeta_{+}(t)}-\delta_{\zeta_{-}(t)}\right] \mathscr{F}_{t} .
$$

From Eq. (34a) with Euler index $2 m$, we have

$$
\delta_{\xi}=-£_{\xi}+\Delta\left(\tilde{\mathfrak{f}} 2 m \hat{z}^{a}\right),
$$

where we may take $\mathfrak{f}=0$ for $r \leqq 2 l, \mathfrak{f}=1$ for $r \geqq 2 l+\varepsilon$. From Eq. I(56) (which holds for $\mathscr{F}$ satisfying (13) and (16b)),

$$
\delta_{\zeta_{ \pm}(t)}= \begin{cases}-£_{\zeta_{ \pm}(t)} \mathscr{F}+\Delta\left[m \hat{z}^{a}\right] \mathscr{F}, & r_{ \pm}(t) \leqq R+\varepsilon \\ 0, & \text { elsewhere }\end{cases}
$$

where $r_{ \pm}(t)=r-o_{ \pm}(t)$. Equations (21)-(23) imply

$$
\mathscr{F}{ }_{t}(r)= \begin{cases}\underset{\sim}{U}\left(-t m \hat{z}^{a}\right) \mathscr{F}\left(r_{ \pm}(t)+o_{ \pm}\right), & r_{ \pm}(t) \leqq R+\varepsilon \\ \underset{\sim}{U}\left[t \tilde{f}(r) 2 m \hat{z}^{a}\right] \hat{R}(t \hat{z}) \mathscr{F}(r), & \text { elsewhere }\end{cases}
$$

Then, by Eqs. (16)

$$
E \mathscr{F}= \begin{cases}U\left(-2 \pi m \hat{z}^{a}\right) \mathscr{F}(r), & r_{+} \leqq R \\ U\left[\mathfrak{f} 2 \pi m \hat{z}^{a}\right] \mathscr{F}(r), & \text { elsewhere } .\end{cases}
$$

Finally, if $g(r)=[1-\mathfrak{f}(r)] f\left(r_{-}\right)$then, because $g=0$ outside $r=2 l+\varepsilon$,

$$
[E \mathscr{F}]=\left[U\left(g(r) 2 \pi m \hat{z}^{a}\right) E \mathscr{F}\right]
$$

and, renaming $U E \mathscr{F}, E \mathscr{F}$, we have

$$
\begin{aligned}
E \mathscr{F} & = \begin{cases}U\left[f\left(r_{-}\right) 2 \pi m \hat{z}^{a}\right] \mathscr{F}, & r_{-} \leqq R+\varepsilon \\
\mathscr{F}, & \text { elsewhere }\end{cases} \\
& =R_{-}\left(2 \pi m \hat{z}^{a}\right) \mathscr{F} .
\end{aligned}
$$

Proof of Theorem 1. We are to show, for any $\overline{\mathscr{F}} \in \mathscr{C}^{2}{ }_{m}$ whose monopoles are in the same internal configuration, that

$$
\psi(E[\overline{\mathscr{F}}])=(-1)^{m n} \psi([\overline{\mathscr{F}}]) .
$$

Observe first that for given initial monopole positions, one exchange differs from another only by the translation of each monopole about some closed loop. By Eq. I(54), any two exchanges $E$ and $\hat{E}$ are then related by $U(1)$ rotations in the manner

$$
\hat{E}=\underset{\sim}{u_{+}}(\chi) \underset{\sim}{u_{-}}(-\chi) E,
$$


and we have

$$
\psi(\hat{E}[\mathscr{F}])=e^{i n(\chi-\chi)}(E[\mathscr{F}])=\psi(E[\mathscr{F}]) .
$$

That is, all exchanges are equivalent on $\mathscr{H}_{m n}^{N}$.

If the monopoles lie on the $y$-axis, Lemmas $1-3$ imply there is a field $\mathscr{F}$ related to $\overline{\mathscr{F}}$ by $U(1)$ rotations of the monopoles,

$$
[\mathscr{F}]=\underline{\sim}_{+}\left(\chi_{+}\right) \underline{\sim}_{-}\left(\chi_{-}\right)[\overline{\mathscr{F}}] \text {, }
$$

and such that

$$
E[\mathscr{F}]=\underset{\sim}{R}-(2 \pi)[\mathscr{F}] .
$$

Since $\underset{\sim}{R}{ }_{-}(2 \pi)[\mathscr{F}]={\underset{\sim}{u}-}_{-}(m \pi)[\mathscr{F}]$, we have

$$
\psi(E[\mathscr{F}])=(-1)^{m n} \psi([\mathscr{F}]) .
$$

Because $U(1)$ rotations commute with monopole translations,

$$
\begin{aligned}
E[\overline{\mathscr{F}}] & =E{\underset{\sim}{+}}_{+}\left(-\chi_{+}\right) u_{\sim}\left(-\chi_{-}\right)[\mathscr{F}] \\
& ={\underset{\sim}{u}-}_{-}\left(-\chi_{+}\right) u_{+}\left(-\chi_{-}\right) E[\mathscr{F}] \\
& =u_{-}\left(\chi_{-}-\chi_{+}\right) u_{+}\left(\chi_{+}-\chi_{-}\right) \underline{u}_{-}(m \pi)[\overline{\mathscr{F}}] .
\end{aligned}
$$

Therefore

$$
\begin{aligned}
\psi(E[\overline{\mathscr{F}}]) & =\exp \left[\operatorname{in}\left(\chi_{-}-\chi_{+}+\chi_{+}-\chi_{-}+m \pi\right)\right] \psi([\overline{\mathscr{F}}]) \\
& =(-1)^{m n} \psi([\overline{\mathscr{F}}]) .
\end{aligned}
$$

If the monopoles are not on the $y$-axis, they may be first translated to the axis, then exchanged and then translated back. The resulting exchange has the form $T E T^{-1}$; and, since $E$ is a product of U(1)-rotations, it commutes with the translation $T$. Thus $T E T^{-1}=E$ and Eq. (8) holds for this case as well.

In our discussion the basic result (Lemma 4.3) was the fact that for a symmetrically chosen field $\mathscr{F}$, exchange by translation was equal modulo $\mathscr{G}^{\infty}$ to a $2 \pi$-rotation of one of the monopoles. This feature of the exchange can be visualized by looking at the Higgs field as in Fig. 1(a)-(e). ${ }^{4}$

\section{The Asymptotic Fock Space}

a. The Asymptotic $N$-Monopole Configuration Space. The limit $\lambda \rightarrow 0$ in which monopole separation is large compared to the size of each monopole can be

4 In fact it seems evident that the U(1) character of the bundle $\mathscr{C}_{m}^{N}$ originates in the way in which the positions of the monopoles determine the possible configurations of the Higgs field $\Phi^{a}$ and specifically in how $\Phi^{a}$ necessarily contorts as the monopoles move about. To clarify this might result in a derivation of monopole statistics conducted entirely in terms of the Higgs field as envisioned in [8]. However, the claim in that reference that monopoles can display spin-1/2 in the absence of the isospinor field $\sigma$ is erroneous. It appears to result from the unwarranted interpretation of a monopole as a "symmetric top". Such an interpretation is contradicted, for example, by the study of exact monopole solutions in [9], which finds four (three translational plus one phase) degree of freedom for each monopole, not $6=3+3$ as would be the case for a "top" 
realized formally by maintaining the location of the monopoles and shrinking their radius $R$. One can make this idea precise by introducing the direct limit as $R \rightarrow 0$ of the bundles $\mathscr{C}^{N}(R)$, the configuration spaces of $N$ monopoles of radius $\leqq R$. A limiting bundle $\tilde{\mathscr{C}}^{N}$ exists because for $R^{\prime}<R, \mathscr{C}^{N}(R)$ is isomorphic to the sub-bundle of $\mathscr{C}^{N}\left(R^{\prime}\right)$ in which monopole separations are greater than $2 R$ :

Proposition. $\mathscr{C}^{N}(R) \simeq \mathscr{C}^{N}\left(R^{\prime}\right) \backslash\left\{[\mathscr{F}] \in \mathscr{C}^{N}\left(R^{\prime}\right)\right.$ with $\left|o_{\alpha}-o_{\alpha^{\prime}}\right| \leqq 2 R$, some $\left.\alpha, \alpha^{\prime}\right\}$ via a bundle isomorphism that preserves the monopole positions $o_{\alpha}$.

An explicit set $\mathfrak{S}$ of isomorphisms is given in the Appendix.

Definition. $\tilde{\mathscr{C}}^{N}$ is the direct limit of the sequence of bundles $\mathscr{C}^{N}(R)$ with respect to the isomorphisms of $\Xi$. Then $\tilde{\mathscr{C}}^{N}$ is a bundle with fibers isomorphic to $\mathrm{U}(1) \times \ldots \times \mathrm{U}(1)$ and in which the monopoles' locations are constrained only by the requirement that no two monopoles coincide, $o_{\alpha} \neq o_{\alpha}^{\prime}$.

One may similarly introduce the sub-bundles $\tilde{\mathscr{C}}_{m}^{N}$ of $\tilde{\mathscr{C}}^{N}$ :

Definition. $\tilde{\mathscr{C}}_{m}^{N}$ is the direct limit of the sequence of bundles $\mathscr{C}_{m}^{N}(R)$. Because the fibers of $\tilde{\mathscr{C}}_{m}^{N}$ are again isomorphic to $\mathrm{U}(1) \times \ldots \times \mathrm{U}(1)$, asymptotic Hilbert spaces $\mathscr{H}_{m n}^{N}$ representing monopoles of magnetic charge $m g$ and electric charge $n \frac{e}{2}$ can be defined in accordance with Eq. (41) as the functions on $\tilde{\mathscr{C}}_{m}^{N}$ homogeneous of degree $n$ with respect to each $\mathrm{U}(1)$.

Definition. $\tilde{\mathscr{H}}_{m n}^{N}=\left\{\psi \in L^{2}\left(\tilde{\mathscr{C}}_{m}^{N}\right)\right.$ such that the restriction of $\psi$ to any fiber has the form $\psi_{o} e^{i n\left(\chi_{1}+\ldots+\chi_{N}\right)}$, where $\chi_{1}, \ldots, \chi_{N}$ are $N$ phases parametrizing that fiber $\}$.

Remark. In the special case $N=1$, the limiting process is superfluous and $\tilde{\mathscr{C}}^{1}=\mathscr{C}^{1}$, $\tilde{\mathscr{H}}_{m n}^{1}=\mathscr{H}_{m n}$ (strictly, $\mathscr{H}_{m n}^{1}(R)$ for any $R$ ).

The aim of this section is to show that $\tilde{\mathscr{H}}_{m n}^{N}$ is the symmetric or antisymmetric subspace of the tensor product $\mathscr{H}_{m n}^{1} \otimes \ldots \otimes \mathscr{H}_{m n}^{1}$ of $N 1$-monopole Hilbert spaces, when $m n$ is even or odd, respectively.

This characterization of dyon statistics depends ultimately only on the topological (or, more properly, bundle theoretic) character of $\mathscr{C}_{m}^{N}$. In fact the internal monopole degrees of freedom play an inessential role in determining dyon statistics and we may begin by studying a smaller bundle from which they have been frozen out. They will be restored in Sect. Ve.

Let us fix, then, a particular internal monopole configuration $\Sigma$ and consider the sub-bundle $B$ of $\mathscr{C}_{1}^{2}$ consisting of pairs of identical monopoles each with unit magnetic charge, and internal configuration $\Sigma$. This restricted configuration space $B$ has fibers isomorphic to $\mathrm{U}(1) \times \mathrm{U}(1)$, and the isomorphism is unique up to an action of $\mathrm{U}(1) \times \mathrm{U}(1)$ or an exchange of the two U(1)'s. ${ }^{5}$ The base space $M$ of $B$ consists in effect of unordered pairs of distinct points of $\mathbb{R}^{3}$, each specifying the location of one of the monopoles, the point where the Higgs field vanishes.

b. The Labelled-Monopole Bundle and Its Exchange Automorphism. To help determine the structure of $B$ let us introduce a second bundle $\bar{B}$, obtained from $B$ by "labelling" the monopoles. In other words a point of $\bar{B}$ is a configuration $\mathscr{F}$ in $B$ augmented by an arbitrary ordering of the unordered pair of monopole centers.

5 The group of the bundle $B$ is thus not $U(1) \times U(1)$ but its semi-direct product with $\mathbb{Z}_{2}$ 
Thus $\bar{B}$ has base space

$$
\bar{M}=\mathbb{R}^{3} \times \mathbb{R}^{3} \backslash \operatorname{diag}=\left\{\left(o_{1}, o_{2}\right) \in \mathbb{R}^{3} \times \mathbb{R}^{3} \mid o_{1} \neq o_{2}\right\}
$$

and is formally the pullback, ${ }^{6} \overleftarrow{t}(B)$, of $B$ over the projection $t: \bar{M} \rightarrow M$ which takes the ordered pair $\left(o_{1}, o_{2}\right) \in \bar{M}$ into the unordered pair $\left\{o_{1}, o_{2}\right\} \in M$.

The advantage of introducing $\bar{B}$ is that in it the $\mathrm{U}(1)$ acting on the "first" monopole does not get confused with that acting on the "second" monopole. In consequence $\bar{B}$ admits a globally defined action of $\mathrm{U}(1) \times \mathrm{U}(1)$ :

$$
\overline{\mathscr{F}} \rightarrow \Gamma\left(u_{1}, u_{2}\right) \mathscr{F},
$$

$u_{1}, u_{2} \in \mathrm{U}(1), \overline{\mathscr{F}} \in \bar{B}$. Thus $\bar{B}$ is a principal fiber bundle. Moreover, the parallel transport defined on $\mathscr{C}^{N}(R)$ by Eqs. I(53) commutes to leading order in $\lambda$ with the set $\mathfrak{G}$ of isomorphisms $\mathscr{C}^{N}(R) \rightarrow \mathscr{C}^{N}\left(R^{\prime}\right)$. It therefore induces on $\bar{B}$ an exact connection whose curvature is specified by giving the $U(1)$ transformation that corresponds to vectors $\left(n_{1}^{i}, n_{2}^{i}\right)$ and $\left(\bar{n}_{1}^{i}, \bar{n}_{2}^{i}\right)$ in the base space $\bar{M}$. That is, the result of translating $o_{1}$ around an infinitesimal loop in $\bar{M}$ spanned by these vectors is, from Eq. I(54), the transformation $\Gamma\left(u_{1}, u_{2}\right)$, where

$$
u_{1}=-u_{2}=\frac{1}{2} \stackrel{o}{F}_{i j}\left(n_{1}{ }^{i} \bar{n}_{1}^{j}-n_{1}{ }^{i} \bar{n}_{2}^{j}-n_{2}{ }^{i} \bar{n}_{1}{ }^{j}+n_{2}{ }^{i} \bar{n}_{2}^{j}\right) \text {, }
$$

with $\stackrel{o}{F}_{i j}=\varepsilon_{i j k}\left(o_{1}{ }^{k}-o_{2}{ }^{k}\right) /\left|o_{1}-o_{2}\right|^{3}$.

A principal bundle with connection will be called a gauge space.

Let us define further $\Gamma_{1}(u)=\Gamma(u, 1), \Gamma_{2}(u)=\Gamma(1, u)$, which explicitly "untangles" the 2 U(1)'s. By ignoring the phase associated with the second (first) monopole we obtain from $\bar{B}$ a U(1) gauge space $B_{1}\left(B_{2}\right)$ acted on by $\Gamma_{1}\left(\Gamma_{2}\right)$. Formally $B_{1}$ is the quotient of $\bar{B}$ with respect to the action of $\Gamma_{2}$ and vice versa:

$$
B_{1}=\bar{B} / \Gamma_{1}, \quad B_{2}=\bar{B} / \Gamma_{2} .
$$

Thus a point $\overline{\mathscr{F}} \in \bar{B}$ gives rise to a pair of points $\mathscr{F}_{i} \in B_{i}$, and conversely it is clear that any such pair determines a unique point of $\bar{B}$. In other words, $\bar{B}$ (including the connection) is the fiber product ${ }^{7}$

$$
\bar{B}=B_{1} \times B_{2},
$$

and its study, thereby reduces to that of a pair of simpler structures - U(1)-gauge spaces - over $\bar{M}$.

Let us study $B_{1}$. By definition, its connection prescribes how the phase of the "first" monopole changes under arbitrary translations of both monopoles. From (28), the curvature 2 -form of $B_{1}$ is

$$
\operatorname{curv}\left(B_{1}\right)=\frac{1}{2} \stackrel{o}{F}_{i j}\left(y_{12}\right) d y_{12}{ }^{i} \otimes d y_{12}^{j},
$$

6 "Pullback" is defined, e.g., in [5]

7 The fiber product of bundles $B_{1}, B_{2}$ over $M$ is the bundle $B$ whose fiber over any point $x$ is the product of the fibers over $x$ of $B_{1}$ and $B_{2}$. We have in effect merely used that when the group of a gauge space is a product, $G=G_{1} \times G_{2}$, the space itself is the fiber product of gauge spaces with groups $G_{1}, G_{2}$ 
where $y_{12}=o_{1}-o_{2}$ and $\stackrel{o}{F}_{i j}(y)=\frac{\varepsilon_{i j k} y^{k}}{|y|^{3}}$ (recall that $m=1$ ). As shown in [5], this uniquely determines both the bundle structure and the connection of $B_{1}$ to be those of the pullback over $y_{12}$ (regarded as the map $\left.\left(o_{1}, o_{2}\right) \rightarrow y_{12}\right)$ of the "dyonic" gauge space $\mathbb{D}_{1 / 2}$ defined in Appendix A of [5], the center of mass configuration space of a Dirac-dyon for which $Q_{E} Q_{B}=\frac{1}{2}$,

$$
B_{1} \simeq \overleftarrow{y}_{12}\left(\mathbb{D}_{1 / 2}\right)
$$

By symmetry we have also

$$
B_{2} \simeq \overleftarrow{y}_{21}\left(\mathbb{D}_{1 / 2}\right)
$$

Now to each point of $B$ there correspond exactly two points of $\bar{B}$. Hence the exchange of such points is an automorphism $P: \bar{B} \rightarrow \bar{B}$ and $B$ itself is the quotient of $\bar{B}$ with respect to $P$. By determining $P$ as an automorphism of $\overleftarrow{y}_{12}\left(\mathbb{D}_{1 / 2}\right) \times \overleftarrow{y}_{21}\left(\mathbb{D}_{1 / 2}\right)$, we determine both the bundle structure of $B$ and its connection.

Now by definition (see [5], Appendix B), an element of $\overleftarrow{y}_{12}\left(\mathbb{D}_{1 / 2}\right)$ in the fiber over $\left(o_{1}, o_{2}\right)$ is a triple $\left(\xi, o_{1}, o_{2}\right)$ such that $\pi_{1 / 2}(\xi)=o_{1}-o_{2}$, where $\pi_{1 / 2}: \mathbb{D}_{1 / 2}$ $\rightarrow \mathbb{R}^{3} \backslash\{0\}$ is the defining projection of $\mathbb{D}_{1 / 2}$. An element of $\overleftarrow{y}_{21}\left(\mathbb{D}_{1 / 2}\right)$ is a triple $\left(\eta, o_{1}, o_{2}\right)$ with $\pi_{1 / 2}(\eta)=o_{2}-o_{1}$. Hence, by (29) and (32), an element of $\bar{B}=B_{1} \times B_{2}$ in the fiber over $\left(o_{1}, o_{2}\right)$ can be identified with a quadruple $\left(\xi, \eta, o_{1}, o_{2}\right)$ such that

$$
\pi_{1 / 2}(\xi)=-\pi_{1 / 2}(\eta)=o_{1}-o_{2} .
$$

With respect to this identification let us write $P\left(\xi, \eta, o_{1}, o_{2}\right)=\left(\xi^{\prime}, \eta^{\prime}, o_{2}, o_{1}\right)$, noting that $P$ exchanges the fiber over $\left(o_{1}, o_{2}\right)$ with that over $\left(o_{2}, o_{1}\right)$. Then from

$$
\pi_{1 / 2}\left(\xi^{\prime}\right)=\left(o_{2}-o_{1}\right)=\pi_{1 / 2}(\eta),
$$

it follows that $\xi^{\prime}=u_{1} \eta$ for $u_{1} \in \mathrm{U}(1)$; similarly $\eta^{\prime}=u_{2} \xi$. Because $P$ preserves the connection on $\bar{B}, u_{1}$, and $u_{2}$ must be overall constants. And because $P$ is by definition an exchange of points in $\bar{B}$, we have $P^{2}=1$, or $u_{1} u_{2}=1$. Finally, we can use the overall phase freedom in the identifications (32) to arrange that $u_{1}$ (and therefore $u_{2}$ ) be 1 . We thus obtain $P$ in the simple form

$$
\left(\xi, \eta, o_{1}, o_{2}\right) \leftrightarrow\left(\eta, \xi, o_{2}, o_{1}\right) .
$$

For future reference we define $P_{12}: B_{2} \rightarrow B_{1}$ and $P_{21}: B_{1} \rightarrow B_{2}$ by

$$
P_{12}\left(\xi, o_{1}, o_{2}\right)=\left(\xi, o_{2}, o_{1}\right) ; \quad P_{21}=P_{12}^{-1} \text {. }
$$

Then $P=P_{21} \times P_{12}$.

c. Exchange of Minimally Charged Identical Dyons with Frozen Internal Dynamics. A wave function $\psi$ in $\tilde{\mathscr{H}}_{1,1}^{1}$ with support contained in the bundle $B$ defined above describes a quantum state in which two monopóles of electric charge $\frac{e}{2}$ and magnetic charge $\frac{-1}{e}$ are present and in which zero amplitude is assigned to any $\psi$ whose monopoles have internal configurations other than $\Sigma$. We want to show that the set $\hat{\mathscr{H}}$ of all such $\psi$ is naturally identified with the set of complex valued functions on $\mathbb{R}^{3} \times \mathbb{R}^{3}$ odd with respect to exchange of arguments. 
To this end, notice first that, in light of the preceding section, any element $\psi: B \rightarrow C$ of $\hat{\mathscr{H}}$ can be constructed as a function on $\bar{B}=B_{1} \times B_{2}$ obeying

$$
\psi\left(\xi, \eta, o_{1}, o_{2}\right)=\psi\left(\eta, \xi, o_{2}, o_{1}\right)
$$

and homogeneous in the sense that

$$
\begin{aligned}
& \psi\left(u \xi, \eta, o_{1}, o_{2}\right)=u \psi\left(\xi, \eta, o_{1}, o_{2}\right), \\
& \psi\left(\xi, u \eta, o_{1}, o_{2}\right)=u \psi\left(\xi, \eta, o_{1}, o_{2}\right) .
\end{aligned}
$$

Because of its joint homogeneity on $B_{1} \times B_{2}, \psi$ can be further reinterpreted as a function on the gauge-space [5] $B_{1} \otimes B_{2}$, whose fibers are those of $B_{1} \times B_{2}$ modulo the equivalence relation $(u \xi, \eta) \sim(\xi, u \eta)$. (We write the equivalence class of $(\xi, \eta)$ as $\xi \otimes \eta$.) This gives $\hat{\mathscr{H}}$ as the space

$$
\left\{\psi \in L_{h}^{2}\left(B_{1} \times B_{2}\right) \mid \psi \circ \Pi=\psi\right\}
$$

of homogeneous wave functions on $B_{1} \times B_{2}$, invariant under the inversion $\Pi \in \operatorname{Aut}\left(B_{1} \times B_{2}\right)$ defined by

$$
\Pi=P_{21} \otimes P_{12}
$$

or explicitly,

$$
\Pi\left(\xi \otimes \eta, o_{1}, o_{2}\right)=\left(\eta \otimes \xi, o_{2}, o_{1}\right) .
$$

But in [5] precisely this situation occurred, with $B_{1} \otimes B_{2}$ denoted by ' $E_{0}^{\mathrm{res}}(1,2)$ ', and $\Pi$ denoted by ' $\Pi_{o}^{\text {res }}(P)^{8}$. Equation (3.42) of that reference established that $B_{1} \otimes B_{2}$ is in fact a trivial gauge-space:

$$
B_{1} \otimes B_{2} \simeq \mathrm{U}(1) \times \bar{M} ;
$$

and the proof of Theorem 3 there established that with respect to the presentation (37), $\Pi$ takes the form

$$
\Pi=(-1) \times \varpi,
$$

where $\varpi\left(o_{1}, o_{2}\right)=\left(o_{2}, o_{1}\right)$. As in [5] it follows immediately from this that $\hat{\mathscr{H}}$ is equivalent to the space of $L^{2}$ functions $\psi\left(o_{1}, o_{2}\right)$ which are odd under interchange of arguments:

$$
\hat{\mathscr{H}} \simeq L^{2}\left(\mathbb{R}^{3}\right) \wedge L^{2}\left(\mathbb{R}^{3}\right) .
$$

In other words, dyons of minimum electric and magnetic charge are fermions.

d. Exchange for General Electric and Magnetic Charge. At least they behave like fermions when their internal dynamics have been frozen. Before indicating how to handle the internal degrees of freedom, let us dispose of the restriction to minimum electric and magnetic charge. (We also restricted to $N=2$, but the extension to arbitrary $N$ is mechanical, given the work of [5], and in particular, Theorem 3 , therein.)

8 To see that $E_{o}^{\text {res }}$ is our $B_{1} \otimes B_{2}$, compare with Eqs. (3.38) (with $n=1$ ) and (2.19) of [5]. To see that $\Pi_{o}^{\text {res }}(P)$ is our $\Pi$, compare with the first equation on p. 182 [whose notation is explained following Eq. (2.35)] and notice that therein $P$ is the exchange $(1 \leftrightarrow 2)$. In [5] the condition $\psi=\psi \circ \Pi$ had the significance that the individual $e$-poles and $g$-poles act like bosons 
For monopoles of Euler index $m=1\left(Q_{B}=-\frac{m}{e}\right)$, the curvature 2-form in (81) would be multiplied by $m$, whose only effect on the subsequent analysis would be to put $\mathbb{D}_{m / 2}$ in place of $\mathbb{D}_{1 / 2}$, and therefore (since $\mathbb{D}_{m / 2}$ is the quotient of $\mathbb{D}_{1 / 2}$ by $\sqrt[m]{1}$ in $\mathrm{U}(1)$ ), to replace (34) by the condition that $\psi$ be homogeneous of degree $m$ in $u$. Similarly a $\psi \in \mathscr{H}_{m n}$ is by definition homogeneous of degree $n$ on the relevant configuration bundle and therefore equivalent, from what has just been said, to a function of degree $m n$ on the bundle $\bar{B}$ of the previous subsection. Consequently $\Pi$ in $(37 b)$ would induce a sign $(-1)^{m n}$ in $\psi$, whence the fermionic dyons are precisely those with $m n$ odd.

Remarks. The above discussion has formulated the question of monopole statistics in terms of the structure of a space $\hat{\mathscr{H}}$ of wave functions which, because of their double homogeneity on $B$ could have been regarded as defined on a bundle $\hat{B}$ over $M$ whose fibers are simple U(1)'s. ( $\hat{B}$ is the quotient of $B_{1} \times B_{2}$ by $\Pi$.) Now it is known that $M$ (which is homotopic to $\mathbb{P}^{2}$ ) admits only 2 distinct $\mathrm{U}(1)$-bundles, and further that a choice of one of these amounts to a choice of the bose or fermi character of the elements of $\hat{\mathscr{H}}=L_{h}^{2}(\hat{B})[10]$. To determine dyon statistics then, is merely to determine which equivalence class of $U(1)$ bundles $\hat{B}$ belongs to, and we have in fact determined this class in two independent ways, both resting on our introduction in Paper I of a notion of monopole transport. The way followed in this section has used aside from general properties of U(1) bundles only the curvature of the bundle's connection. Since this curvature only expresses the effect of carrying an electric charge around a closed path in an external magnetic field, the same arguments should hold for monopoles in any gauge theory whose unbroken symmetry group reduces to $\mathrm{U}(1)$.

e. Restoring the Internal Degrees of Freedom. Returning to the full asymptotic bundle $\tilde{\mathscr{C}}_{m}^{N}$ and to the corresponding state spaces $\tilde{\mathscr{H}}_{m n}^{N}$, we can now establish the main result of this section.

Theorem 2. The space $\tilde{\mathscr{H}}_{m n}^{N}$ is the totally symmetric (antisymmetric) subspace of the $N$-fold tensor product of the 1-dyon state-spaces

$$
\tilde{\mathscr{H}}_{m n}^{1} \otimes \ldots \otimes \tilde{\mathscr{H}}_{m n}^{1},
$$

when $m$ is even (odd). Moreover,

$$
\tilde{\mathscr{H}}_{m n}^{1}=\tilde{\mathscr{H}}_{m n}^{1^{\text {int }}} \otimes L^{2}\left(\mathbb{R}^{3}\right),
$$

where the two factors represent the internal and external degrees of freedom of a monopole in $\tilde{\mathscr{H}}_{m n}^{1}$.

Equation (40) shows that the decomposition (39) is physically natural, at least on a kinematical level ${ }^{9}$. It will also enable us to define dyon spin in the present

9 However, any kinematic requirement is physically incomplete without a further dynamical condition guaranteeing "sufficiently weak interactions" among particles. By this phrase, which we do not attempt to make precise, we mean to suggest the stability and relative independence needed for the notion of particle to be physically meaningful. In particular, a mutual decoupling of particles at large separations is needed for statistics to have the correct consequences in scattering; and rough reduction of interactions to a (for example 2-particle) form independent of the number of particles actually present is needed, e.g., in statistical mechanics 
language ${ }^{10}$, and, incidentally, it allows the introduction in $\tilde{\mathscr{H}}_{m n}=\bigoplus_{N} \tilde{\mathscr{H}}_{m n}^{N}$ (but not in all of $\tilde{\mathscr{H}}$ !) of effective creation and annihilation operators for dyons (at least for slow moving ones).

Having seen already how to pass from $m=n=1$ to general $m$ and $n$, we will establish (39) explicitly only for the case $N=2, m=n=1$. That is, we will find a representation of $\tilde{\mathscr{H}}_{1,1}^{2}$ as the antisymmetric subspace of $\tilde{\mathscr{H}}_{1,1}^{1} \otimes \tilde{\mathscr{H}}_{1,1}^{1}$ :

$$
\tilde{\mathscr{H}}_{1,1}^{2} \simeq \tilde{\mathscr{H}}_{1,1}^{1} \wedge \tilde{\mathscr{H}}_{1,1}^{1} .
$$

Once can arrive at (41) by steps closely paralleling those by which its special case (38) was reached. The role of $B$ will be played by the space $\underset{\sim}{B}:=\tilde{\mathscr{C}}_{1}^{2}$, of all $m=1$ (asymptotic) 2-monopole configurations, regarded as a bundle over $M$, the space of monopole locations. When $\mathscr{C}_{1}^{2}$ was regarded as a bundle over $\mathscr{M}_{1}^{2}$, the space of monopole locations and internal configurations its fibers were isomorphic to $U(1) \times U(1)$. However $\underset{\sim}{B}$ 's fibers will be isomorphic to the enlarged space $\mathscr{I} \times \mathscr{I}$, where

$$
\mathscr{I}=\left.\mathscr{C}_{1}^{1}\right|_{o_{1}=0}
$$

is the configuration space of a single monopole of fixed location.

To see this let $\left[\mathscr{F}_{1}\right],\left[\mathscr{F}_{2}\right]$ represent elements of $\mathscr{I}$ and $\Phi=B\left(\left\{o_{1}, o_{2}\right\}\right)$ a fiber of $B$. To $\left[\mathscr{F}_{1}\right]$ corresponds a unique internal configuration of the monopole at $o_{1}$ and similarly for $\left[\mathscr{F}_{2}\right]$, whence $\left[\mathscr{F}_{1}\right]$ and $\left[\mathscr{F}_{2}\right]$ determine an $[\mathscr{F}]$ of $\Phi$ which is unique up to U(1)-rotations of the two monopoles (Lemma 3.1). Moreover once the $U(1) \times U(1)$ ambiguity is resolved for a single pair $\left[\mathscr{F}_{1}\right],\left[\mathscr{F}_{2}\right]$ the correspondence of any other such pair to an element of $\Phi$ becomes unique (because a single pair of $\mathrm{U}(1)$ choices - equivalently a choice of gauge in the intermonopole region I suffices to determine uniquely a correspondence between $\left.\mathscr{F}_{1}\right|_{\beta_{o}}$ and $\left.\mathscr{F}_{2}\right|_{\beta_{o}}$ on the one hand and $\left.\mathscr{F}\right|_{\beta_{1} \cup \beta_{2}}$ on the other, where $\left.\left[\mathscr{F}_{i}\right] \in \tilde{\mathscr{C}}_{1}^{1}(R)\right|_{o_{1}=0},\left.[\mathscr{F}] \in \mathscr{C}_{1}^{2}(R)\right|_{o_{1} o_{2}}$ and $\beta_{0}$ is the ball of radius $R$ about the origin.) Thus we have defined a correspondence

$$
\underset{\sim}{B}\left(\left\{o_{1}, o_{2}\right\}\right) \leftrightarrow \mathscr{I} \times \mathscr{I},
$$

and shown that the freedom therein is a pair of $U(1)$ 's together with a $\mathbb{Z}_{2}$ (giving the ordering of $o_{1}, o_{2}$ ). Since an interchange of $o_{1}$ and $o_{2}$ interchanges the role of these $U(1)$ factors, the group $G$ expressing the freedom in (42) is (with (S) being semi-direct product)

$$
G=\mathrm{U}(1) \times \mathrm{U}(1)\left(\mathbb{Z}_{2} .\right.
$$

In sum $\underset{\sim}{B}$ is a bundle over $M$ with fiber $\mathscr{I} \times \mathscr{I}$ and group $\mathrm{U}(1) \times \mathrm{U}(1)\left(\$ \mathbb{Z}_{2}\right.$. Now to determine any bundle of known fiber it suffices to give an associated bundle with the same base space and group. But the previously defined bundle $B$

10 In the non-relativistic, quantum mechanical context of [5], we asserted that the notion of spin necessarily implies a decomposition of the form (13). In a relativistic context, this claim breaks down because massless (but not spinless) particles have no position observables, whence their spin can be defined only for momentum eigenstates. However, monopoles are not massless and the decomposition (17) does exist, making it possible to study monopole spin without having to investigate how the full Poincaré group is represented on $\mathscr{H}_{m n}^{1}$ 
(for any choice of frozen internal configuration $\Sigma$ ) is a sub-bundle of $\underset{\sim}{B}$ and is associated to it. Hence $\underset{\sim}{B}$ can be characterized as the bundle with fiber $\mathscr{I} \times \mathscr{I}$ associated to $B$.

Using this characterization one can repeat the reasoning of subsections $b$ and c, replacing at each step the relevant bundle with fiber $U(1) \times U(1)$ [respectively $\mathrm{U}(1)$ or $\mathrm{U}(1) \otimes \mathrm{U}(1)=\mathrm{U}(1)]$ by the associated bundle with fiber $\mathscr{I} \times \mathscr{I}$ [respectively $\mathscr{I}$ or $\mathscr{I} \otimes \mathscr{I}$, where, as before, $\mathscr{I} \otimes \mathscr{I}$ is $\mathscr{I} \times \mathscr{I}$ modulo the equiva-

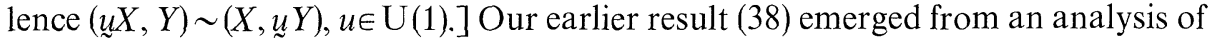
$B$ 's structure culminating in Eqs. $(37 \mathrm{a}, \mathrm{b})$. Expressed in a suitable form for generalization it stated that the homogeneous functions on $B$ correspond to the homogeneous functions on $\mathrm{U}(1) \otimes \mathrm{U}(1) \times \bar{M}$ which change sign under the action of $\sigma \times \varpi$, where $\sigma$ interchanges the $\mathrm{U}(1)$-factors. (Of course $\mathrm{U}(1) \otimes \mathrm{U}(1)=\mathrm{U}(1)$ and $\sigma=1$.) Here the generalization will be that the space $\tilde{\mathscr{H}}_{1,1}^{2}=L_{h}^{2}(B)$ of homogeneous functions on $\underset{\tilde{B}}{B}=\tilde{\mathscr{C}}_{1}^{2}$ corresponds to the space of homogeneous functions on $\mathscr{I} \otimes \mathscr{I} \times \bar{M}$ which change sign under the action of $\underset{\sim}{\sigma} \times \varpi$, where $\sigma(X \otimes Y)=Y \otimes X$.

To interpret this we must put $L_{h}^{2}(\mathscr{I} \otimes \mathscr{I} \times \bar{M})$ in slightly different form. [The corresponding earlier step was trivial:

$$
\left.L_{h}^{2}(\mathrm{U}(1) \otimes \mathrm{U}(1) \times \bar{M})=L_{h}^{2}(\mathrm{U}(1) \otimes \mathrm{U}(1)) \otimes L_{h}^{2}(\bar{M})=L^{2}(M) .\right]
$$

We have (cf. [5], Appendix B, part c)

$$
\begin{gathered}
L_{h}^{2}(\mathscr{I} \otimes \mathscr{I} \times \bar{M})=L_{h}^{2}(\mathscr{I} \otimes \mathscr{I}) \otimes L^{2}(\bar{M}), \\
L_{h}^{2}(\mathscr{I} \otimes \mathscr{I})=L_{h}^{2}(\mathscr{I}) \otimes L_{h}^{2}(\mathscr{I}), \\
L^{2}(\bar{M})=L^{2}\left(\mathbb{R}^{3} \times \mathbb{R}^{3}\right)=L^{2}\left(\mathbb{R}^{3}\right) \otimes L^{2}\left(\mathbb{R}^{3}\right),
\end{gathered}
$$

where the last line used that the complement of $\bar{M}$ is of measure zero in $\mathbb{R}^{3} \times \mathbb{R}^{3}$. Hence $\tilde{\mathscr{H}}_{1,1}^{2}$ corresponds to those elements of

$$
L_{h}^{2}(\mathscr{I}) \otimes L_{h}^{2}(\mathscr{I}) \otimes L^{2}\left(\mathbb{R}^{3}\right) \otimes L^{2}\left(\mathbb{R}^{3}\right)
$$

which change sing under the simultaneous permutation of factors $1 \leftrightarrow 2$ (from $\sigma$ ) and $3 \leftrightarrow 4$ (from $\varpi$ ). Rearranging we find finally

$$
\tilde{\mathscr{H}}_{1,1}^{2} \simeq\left[L_{h}^{2}(\mathscr{I}) \otimes L^{2}\left(\mathbb{R}^{3}\right)\right] \wedge\left[L_{h}^{2}(\mathscr{I}) \otimes L^{2}\left(\mathbb{R}^{3}\right)\right] .
$$

f. The One-particle State-space; Dyon Spin. To complete the proof that $m=n=1$ dyons are fermions it remains only to verify that $L_{h}^{2}(\mathscr{I}) \otimes L^{2}\left(\mathbb{R}^{3}\right)$ is indeed the 1 -dyon state space, $\tilde{\mathscr{H}}_{1,1}^{1}$. This will establish Theorem 2 with $\tilde{\mathscr{H}}_{1,1}^{1}$ int identified as $L_{h}^{2}(\mathscr{I})$ :

$$
\tilde{\mathscr{H}}_{1,1}^{1}{ }^{\text {int }}=L_{h}^{2}(\mathscr{I})
$$

The correct relation between spin and statistics then follows from the fact, which below we interpret the present language, that $\tilde{\mathscr{H}}_{m n}^{1 \text { int }}$ carries half-odd spin iff $m n$ is odd.

To begin with notice that $\tilde{\mathscr{C}}_{1}^{1}$, regard as a bundle over $\mathbb{R}^{3}$ with fiber $\mathscr{I}$, is trivial since its base-space is contractible:

$$
\tilde{\mathscr{C}}_{1}^{1} \simeq \mathscr{I} \times \mathbb{R}^{3} .
$$


Hence $\tilde{\mathscr{H}}_{1,1}^{1}=L_{h}^{2}\left(\tilde{\mathscr{C}}_{1}^{1}\right) \simeq L_{h}^{2}(\mathscr{I}) \otimes L^{2}\left(\mathbb{R}^{3}\right)$ as required. For general $m, n$ we have then

$$
\tilde{\mathscr{H}}_{m n}^{1} \simeq \mathscr{H}_{m n}^{1 \text { int }} \otimes L^{2}\left(\mathbb{R}^{3}\right),
$$

where $\tilde{\mathscr{H}}_{m n}^{1 \text { int }}$ is the space of wave functionals homogeneous of degree $n$ on

$$
\mathscr{I}_{m}=\left.\tilde{\mathscr{C}}_{m}^{1}\right|_{o_{1}=0} \text {. }
$$

Given the decomposition (46) we can define dyon internal angular momentum by the action of spatial rotations on $\tilde{\mathscr{H}}_{m n}^{1 \text { int }}$, which in turn comes from their action on $\mathscr{I}_{m}$. If $R: \overline{\mathrm{SO}(3)} \rightarrow \operatorname{Aut}\left(\mathscr{I}_{m}\right)$ is this latter action, then the $2 \pi$-rotation in $\overline{\mathrm{SO}(3)}$ is always the U(1)-rotation $u(\pi m)$. Hence $L_{h}^{2}(R)$, the induced representation of $\overline{\mathrm{SO}(3)}$ on $\tilde{\mathscr{H}}_{m 1}^{1}$ int , takes the $2 \pi$-rotation into $(-1)^{m}$. Consequently the induced representation on $\tilde{\mathscr{H}}_{m n}^{1 \text { int }}$, takes the $2 \pi$-rotation to $\left[(-1)^{m}\right]^{n}=(-1)^{m n}$.

Remark. In a region of Higgs vacuum surrounding a monopole, the $\mathrm{U}(1)$ rotations of that monopole are global symmetries of the "electromagnetic" U(1) bundle defined by the Higgs field. From this alone it follows [12] that $R(2 \pi)=\underset{\sim}{u}(\pi m)$, where $m$ is the twist-number (and therefore the magnetic charge) of the monopole. Thus the correspondence of odd $m n$ with half-odd spin will hold not only for SU(2) but for any choice of gauge group.

Theorem 2 establishes that dyons behave like familiar particles with welldefined fermi or bose statistics, at least when all dyons present are widely separated and of a single species. Of these two limitations the first is essentially a matter of convenience because a decomposition as in Theorem 2 could also be carried out for general monopole separations. However no decomposition of that form will be possible if dyons of different $m: n$ ratios are present; for then no pathindependent notion of dyon-transport exists [as in fact follows from I(54a) and $\mathrm{I}(45)]^{11}$. Rather dyons in this general setting do not possess any statistics in the customary sense based on tensor product decompositions like (41).

Nevertheless one can define a meaningful dyon statistics by reference to the bundle structure of the set of "position eigenvectors" - vectors $\psi \in \mathscr{H}^{N}$ for which the monopole positions are "sharp" [11]. The generalized notion of statistics which emerges also allows one to see in a more general context why it is that (in three spatial dimensions) bosons can sometimes combine to form fermions, and assures one that in all such cases the spin-statistics connection will hold for the composites if it does for their constituents $[11]^{12}$.

\section{Appendix}

Proposition. For $R \geqq R^{\prime} \quad \mathscr{C}^{N}(R) \simeq \mathscr{C}^{N}\left(R^{\prime}\right) \backslash\left\{[\mathscr{F}] \in \mathscr{C}^{N}\left(R^{\prime}\right)\right.$ with $\left|o_{\alpha}-o_{\alpha^{\prime}}\right| \leqq 2 R$, some $\left.\alpha, \alpha^{\prime}\right\}$ via a bundle isomorphism that preserves the monopole positions $o_{\alpha}$.

11 Hence effective dyon creation operators fulfilling the usual bose or fermi exchange relations do not exist. To obtain effective fields describing dyons one might try using path-dependent operators, as in [13]. Or one could try to show that the point-dyon theory of [14] correctly described Yang-Mills monopoles in an appropriate limit

12 This applies to the present case if, for example, we regard an $m=n=1$ dyon as a bound state of an $m=1, n=0$ dyon with one of the charged $(W$-) bosons of the theory 
Proof. An explicit isomorphism $[\mathscr{I}] \rightarrow[\mathscr{F}]^{\prime}$ is defined as follows. Given $[\mathscr{F}] \in \mathscr{C}^{N}(R)$ choose an $\mathscr{F}$ having in each shell $R \leqq r_{\alpha} \leqq R+\varepsilon$ the form

$$
\left.\begin{array}{rl}
\Phi^{a} & =\hat{x}^{a} \cos m \phi_{\alpha} \sin \theta_{\alpha}+\hat{y}^{a} \sin m \phi_{a} \sin \theta_{\alpha}+\hat{z}^{a} \cos \theta_{\alpha} \\
A^{a}{ }_{i} & =\varepsilon^{a}{ }_{b c} \partial_{i} \Phi^{b} \Phi^{c}+\Phi^{a} A_{i},
\end{array}\right\}
$$

where $\left(r_{\alpha}, \theta_{\alpha}, \phi_{\alpha}\right)$ are spherical coordinates about $o_{\alpha}$ and $A_{i}$ is the unique vector potential for $e F^{\mathrm{ext}}{ }_{i j}=\sum_{\alpha^{\prime} \neq \alpha} e F_{i j}^{\alpha^{\prime}}$ with $A_{i} r_{\alpha}{ }^{i}=0$ and $A_{i}=0$ at $r_{\alpha}=0$. Let $r^{\prime}$ be the point with coordinates $\left(\kappa r_{\alpha}, \theta_{\alpha}, \phi_{\alpha}\right)$, where $\kappa=\frac{R^{\prime}}{R}$. Let $[\mathscr{F}]^{\prime}=\left[\mathscr{F}^{\prime}\right]$ with

$$
\mathscr{F}^{\prime}(r)=\mathscr{F}(r), \quad r_{\alpha} \geqq R,
$$

$\mathscr{F}^{\prime}\left(r^{\prime}\right)$ having the form (A1) for $R^{\prime} \leqq r_{\alpha}^{\prime} \leqq R$; and

$$
\left.\begin{array}{rl}
A^{a^{\prime}}{ }_{i}\left(r^{\prime}\right) & =\frac{1}{\kappa}\left(A^{a}{ }_{i}-\Phi^{a} A_{i}\right)(r)+\Phi^{a}(r) A_{i}\left(r^{\prime}\right) \\
\Phi^{a^{\prime}}\left(r^{\prime}\right) & =\Phi^{a}(r) \\
\sigma^{I^{\prime}}\left(r^{\prime}\right) & =\sigma^{I}(r)
\end{array}\right\} r_{\alpha}^{\prime} \leqq R^{\prime} .
$$

To show that $[\mathscr{F}]^{\prime}$ is well defined, let $\bigcup_{\sim} \in \mathscr{G}^{\infty}$ be any gauge transformation for which $U \mathscr{F}$ retains the form (A1) (for all $\alpha$ ). Then in each shell $R \leqq r_{\alpha} \leqq R+\varepsilon$, $U=\exp \left(i \chi_{\alpha} \Phi^{a} \tau_{a}\right)$ for some constant $\chi_{\alpha}$, and we can therefore define $U^{\prime} \in \mathscr{G}^{\infty}$ with $U^{\prime} \mathscr{F}^{\prime}=(U \mathscr{F})^{\prime}$ by

$$
\left.\begin{array}{l}
U^{\prime}(r)=U(r), \quad r_{\alpha} \geqq R ; \\
U^{\prime}(r)=\exp \left(i \chi_{\alpha} \Phi^{a} \tau_{a}\right), \quad R^{\prime} \leqq r_{\alpha} \leqq R ; \\
U^{\prime}\left(r^{\prime}\right)=U(r), \quad r_{\alpha}^{\prime} \leqq R^{\prime} .
\end{array}\right\}
$$

To show that $[\mathscr{F}] \rightarrow[\mathscr{F}]^{\prime}$ is a bundle morphism it suffices to show that if $u_{\alpha}\left(\chi_{\alpha}\right)$ are $\mathrm{U}(1)$ rotations of the monopoles of $\mathscr{F}$,

$$
\left(\prod_{\alpha}{\underset{\sim}{\alpha} \alpha}_{\alpha}\left(\chi_{\alpha}\right)[\mathscr{F}]\right)^{\prime}=\prod_{\alpha}{\underset{\sim}{\alpha}}_{\alpha}\left(\chi_{\alpha}\right)[\mathscr{F}]^{\prime} .
$$

The argument is identical to that just given, but with $U \in \mathscr{G}^{\infty}$ replaced by the operator

$$
U= \begin{cases}1, & r_{\alpha} \geqq R+\varepsilon \\ \exp \left(i \chi_{\alpha} \Phi^{a} \tau_{a}\right), & r_{\alpha} \leqq R+\varepsilon .\end{cases}
$$

Then (A4) gives $U^{\prime}$ with $(\underset{\sim}{U}[\mathscr{F}])^{\prime}=U_{\sim}^{\prime}[\mathscr{F}]^{\prime}=\prod_{\alpha}{\underset{\sim}{\alpha}}_{\alpha}\left(\chi_{\alpha}\right)[\mathscr{F}]^{\prime}$.

Note that the parallel transport defined on $\mathscr{C}^{N}(R)$ by Eqs. I(53) commutes to leading order in $\lambda$ with the isomorphisms $[\mathscr{F}] \rightarrow[\mathscr{F}]^{\prime}$ defined here.

\section{References}

1. Saha, M.N.: Ind. J. Phys. 10, 145 (1936). See also, for example, Barut, A.O.: Proton form factor magnetic charges, and dyonium. Phys. Rev. D 3, 1747 (1971) 
2. 't Hooft, G. : Magnetic monopoles in unified gauge theories. Nuc. Phys. B 79, 276 (1974); Polyakov, A.M. : Particle spectrum in quantum field theory. ZhETF Pis. Red. 20, 430 (1974) (JETP Lett. 20, 194 (1974))

3. Jackiw, R., Rebbi, C.: Spin from isospin in a gauge theory. Phys. Rev. Lett. 36, 1116 (1976); Hasenfratz, P., 't Hooft, G.: Fermion-boson puzzle in a gauge theory. Phys. Rev. Lett. 36, 1119 (1976). See also Strominger, A., Lykken, J.D.: Spin from isospin in SU(5). Phys. Rev. Lett. 44, 1175

4. Barut, A.O.: Spin-statistics connection for dyonium. Phys. Rev. D 10, 2709 (1974); Goldhaber, A.S. : Connection of spin and statistics for charge-monopole composites. Phys. Rev. Lett. 36, 1122 (1976); Leinaas, J.M., Myrheim, J.: On the theory of identical particles. Nuovo Cimento 37B, 1 (1977); Tolkachev, E.A., Tomil'chik, L.M.: Space-reflection operator and the boson-fermion puzzle in a dyonium model. Phys. Lett. 81B, 173 (1979); Friedman, J.L., Sorkin, R.D.: Dyon spin and statistics: A fiber-bundle theory of interacting magnetic and electric charges. Phys. Rev. D 20, 2511 (1979); Aratyn, H.: Z. Phys. C 12, 361 (1982)

5. Friedman, J.L., Sorkin, R.D. : A spin-statistics theorem for composites containing both electric and magnetic charges. Commun. Math. Phys. 73, 161 (1980)

6. Ezawa, Z.F.: Quantum soliton operators for monopoles and fermionization mechanism of dyons. Phys. Lett. 81B, 325 (1979)

7. Finkelstein, D., Rubinstein, J.: Connection between spin, statistics, and kinks. J. Math. Phys. 9, $1762(1968)$

8. Ringwood, G.A., Woodward, L.M.: Monopoles admit spin. Phys. Rev. Lett. 47, 625 (1981)

9. Weinberg, E.J.: Parameter counting for multimonopole solutions. Phys. Rev. D 20, 936 (1979)

10. Souriau, J.-M.: Structure des systemes dynamiques. Paris: Dunod 1970

11. Sorkin, R.D.: Particle statistics in 3 dimensions. Phys. Rev. D27, 1787 (1983)

12. Witten, E.: Dyons of charge $e \theta / 2 \pi$. Phys. Lett. 86B, 283 (1979)

13. Mandelstam, S.: Quantum electrodynamics without potentials. Ann. Phys. 19, 1 (1962)

14. Brandt, R.A., Filippo, N., Zwanziger, D.: Lorentz invariance from classical particle paths in quantum field theory of electric and magnetic charge. Phys. Rev. D 19, 1153 (1979)

Communicated by S.-T. Yau

Received June 18, 1982; in revised form November 2, 1982 
